\title{
Synthesis and Surface Characterization by Raman Spectroscopy of Water-Dispersible ZnS:Mn Nanocrystals Capped with Mercaptoacetic Acid
}

\author{
Ji Eun Kim, Cheong-Soo Hwang, and Sangwoon Yoon \\ Department of Chemistry, Institute of Nanosensor and Biotechnologv, Dankook Chiversity, Jukjeon, Gleonggi $4+8-701$, Korea \\ 'E-mail: cshrangandankookac.kr; sangw'oonaldankook.ac.kr \\ Received March 26,2008
}

Key Words : ZnS nanocrỵstal. Mn-doped nanocrỵstals. Raman spectroscopy. Water-dispersible nanocrỵstals

Semiconductor nanocrystals have been extensively studied due to their size-dependent optical properties and wide applications. ${ }^{1.2}$ Many efforts have been made to develop nanoparticles with superior optical characteristics, e.g. higher quantum yields. more accessible absorption wavelengths. and wider absorption and narrower enission bands. The incorporation of transition metal ions into semiconductor nanocrystals induces radiative recombination of excited electron-hole pair at doping ion sites rather than at surfaces. which shifts photoluminescence wavelength and increases quantum efficiency. ${ }^{3-9}$ Therefore. transition metal-doped seniconductor nanocrystals have great potential applications as a novel material for display and light-enuitting devices.

The functionalization of surfaces is another important aspect of nanoparticle fabrication. ${ }^{\text {ji }}$ In particular, it is important to make nanoparticles water-dispersible for many biological applications. Surfactants play important roles in the control of dispersed particle sizes, optical properties. and solubility by passivating nanocrystal surfaces. ${ }^{11}$ Here. we report on the synthesis and properties of water-dispersible $\mathrm{Mn}^{2-}$-doped $\mathrm{ZnS}$ nanoparticles stabilized by mercaptoacetic acid (ZnS:Mn-MAA). MAA is widely used as a cappung agent in the synthesis of nanoparticles. ${ }^{2}$ MAA is also the sumplest molecule that has two terminal functional groups, a thiol and a carboxylic acid, which can be deprotonated. allowing it to bind to the surfaces of nanoparticles. In this respect. it would be interesting to determine the nature of surface-bound MAA and. in particular. to deternine which functional group is involved in this binding. Here. we present the Raman spectra of MAA adsorbed on the surfaces of $\mathrm{ZnS}$ : Mn nanocrystals and propose a structure for surfacebound MAA based on spectral analyses and density functional theory (DFT) calculations.

\section{Experimental Section}

To synthesize ZnS:Mn-MAA nanoparticles. we employed a modified co-precipitation method used to produce mercaptopropionate (MPA)-capped Mn-doped ZnS nanocrystals. ${ }^{1 \hat{3}}$ Briefly. solutions of $1 \mathrm{M} \mathrm{ZnSO}+(5 \mathrm{~mL}) .0 .1 \mathrm{M}$ $\mathrm{MnSO}_{4}(1.5 \mathrm{~mL})$, and I M MAA $(20 \mathrm{~mL})$ were mixed. and titrated to $\mathrm{pH} 10.3$ with $2 \mathrm{M} \mathrm{NaOH}$. After bubbling the solution with $\mathrm{N}_{2}$ for 30 munutes to purge air. $\mathrm{Na}_{2} \mathrm{~S}$ aqueous solution ( $\mathrm{M}, 4.5 \mathrm{~mL}$ ) was injected quickly and refluxed for
20 hours. The solution color became yellow as the reaction progressed. ZnS:Mn nanocrystals were precipitated by adding ethanol to the mixture and recovered as a white powder by centrifuging. They were subsequently washed and dried in an oven. These crystals were then subjected to X-ray diffraction (XRD, Bruker D5005), high-resolution transmission electron microscopy (HR-TEM, JEOL JEM3010). and Fourier-transform Raman spectroscopy (Bruker. FRA106/S, resolution: $\mathrm{I} \mathrm{cm}^{-1}$ ) for characterization. The $\mathrm{ZnS}$ :Mn-MAA powder was highly soluble in water and was subjected to UV/ris (Perkin-Elmer) and fluorescence (Shimadzu) spectroscopy in aqueous solution.

\section{Results and Discussion}

The optical properties and morphology of the nanocrystals
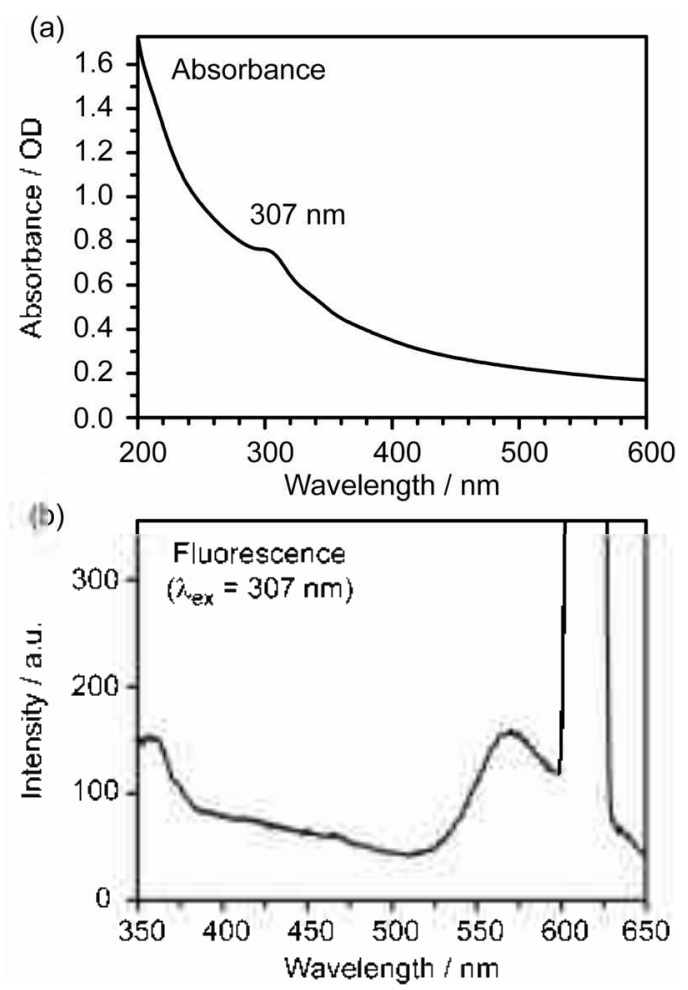

Figure 1. (a) UV-visible absorption spectrum and (b) fluorescence spectrum of MAA-capped ZnS:Mn nanocrystals. The fluorescence spectrum was obtained by excitation at $307 \mathrm{~nm}$. 


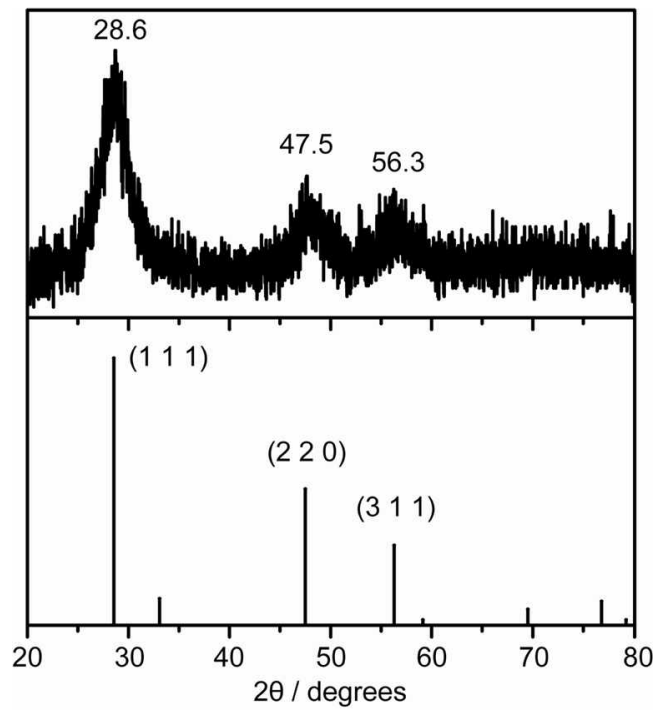

Figure 2. XRD spectrunn of Zn1S:Mn-MAA nanocrystals. The lower panel shows the XRD spectrum of bulk ZnS in a zinc blende form.

produced were determined. Figure I presents the UV/vis and fluorescence spectra of $\mathrm{ZnS}$ :Mn-MAA nanocrystals in water. The absorption spectrum shows a steep increase toward shorter wavelengths with a shoulder at $307 \mathrm{~nm}$. Previously. small absorption bands have been reported at 287 and 290 $\mathrm{nn}$ for other types of Mn-doped $\mathrm{ZnS}$ nanocrystals of size 3 $\mathrm{ln}$ and $3.2 \mathrm{~lm}$. respectively. ${ }^{1 \hat{,}, 14}$ In the present study, a slight red-shift in the absorption band to $307 \mathrm{~nm}$ suggests a particle size larger than $-3 \mathrm{~nm}$. Figure l(b) shows the fluorescence spectrum obtained by exciting at $307 \mathrm{~nm}$. The second-order diffraction of the excitation wavelength appears at $614 \mathrm{~nm}$. Despite the interference of light used for excitation, a fluorescence band is clearly visible with a peak at $\sim 570 \mathrm{~lm}$. The large Stokes shift is attributed to the ${ }^{4} T_{1} \rightarrow{ }^{6} A_{1}$ transition of the $\mathrm{Mn}^{2+}$ doping agent.

The structural properties of $\mathrm{ZnS}: \mathrm{Mn}$ nanocrystals were investigated by XRD and HR-TEM. Figure 2 shows the

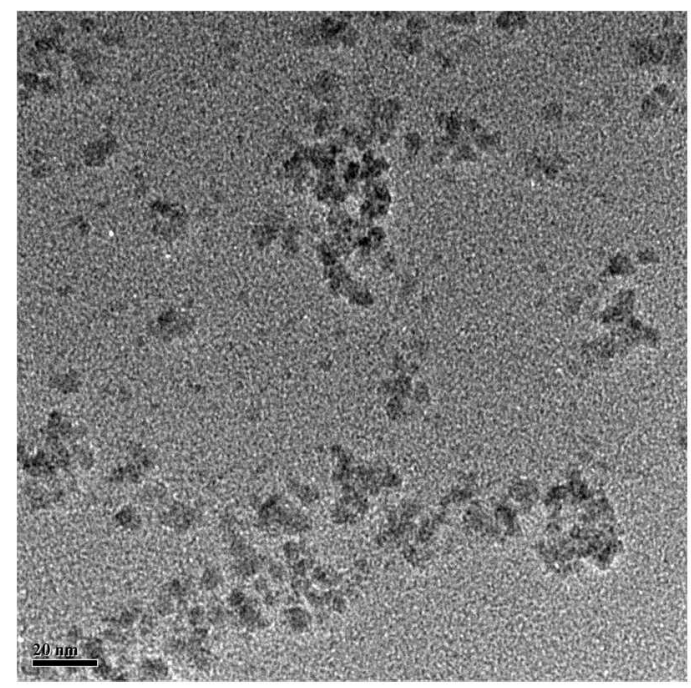

Figure 3. HR-TEM inage of ZnS:Mn-MAA. The scale bar indicates 20 nun.

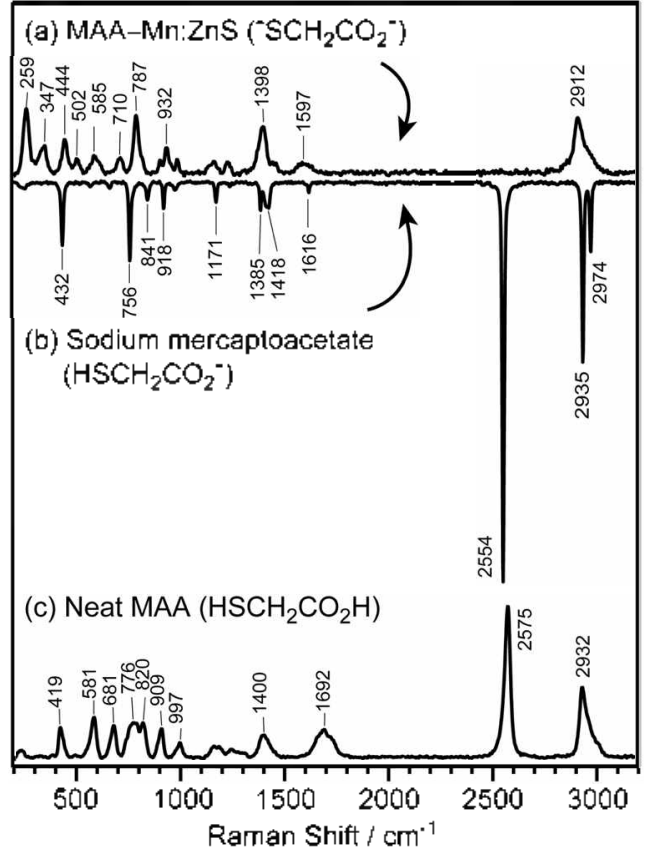

Figure 4. FT-Raman spectra of (a) ZnS:Mn-MAA nanocrystals, (b) sodium mercaptoacetate, and (c) neat MAA solution. All spectra are offet for clarity and the spectrum of sodium mercaptoacetate in (b) is inverted to better allow comparison with (a).

measured XRD pattern of ZnS:Mn-MAA along with that of bulk $\mathrm{ZnS}$. The observed lines at $28.6^{\circ}, 47.5^{\circ}$. and $56.3^{\circ}$ correspond to the (111), (220). and (311) planes of the zincblende form of $\mathrm{ZnS}$. The transmission electron micrograph in Figure 3 shows that the average particle size is $3.8 \pm 0.5$ nim.

The form of the MAA adsorbed on the ZnS:Mn nanoparticles is inferred from Raman spectra. FT-Raman spectra of $\mathrm{ZnS}: \mathrm{Mn}$-MAA nanocry stals were obtained and compared with those of neat MAA solution and sodium mercaptoacetate. $^{15.16}$ The overall spectrum of the $\mathrm{ZnS}: \mathrm{Mn}-\mathrm{MAA}$ nanoparticles was found to closely resemble that of sodium mercaptoacetate except for $2554 \mathrm{~cm}^{-1}$ and low frequency regions, as shown in Figure 4(a) and (b). The absence of S-H stretch at $2554 \mathrm{~cm}^{-1}$ in Figure 4(a) suggests that MAA exists as a thiolate on the nanocrystal surface. In addition. due to deprotonation of carboxylic acid group. the $\mathrm{C}=\mathrm{O}$ stretching band of neat MAA at $1692 \mathrm{~cm}^{-1}$ [Fig. 4(c)] switched to an antisymmetric $\mathrm{OCO}$ stretch at $\sim 1600 \mathrm{~cm}^{-1}$ and a symmetric OCO stretch at $\sim 1400 \mathrm{~cm}^{-1}$ [Fig. 4(b)]. Since the OCO stretch of MAA adsorbed on ZnS:Mn closely matches that of sodium mercaptoacetate. we consider that MAA is adsorbed in a dianionic form and carboxylate is left free as in sodium mercaptoacetate.

Spectral analysis of the low frequency region is rather challenging. MAA/nanoparticle binding restricts low frequency bending. twisting. and torsional motion and shifts these bands considerably compared with those of free MAA. New peaks appear at 259 and $347 \mathrm{~cm}^{-1}$. and these are possibly assigned to transverse and longitudinal optical phonon, respectively. ${ }^{17,18}$ Altematively, DFT calculations [B3LYP/6- 
Table 1. The measured Rannan frequencies of MAA adsorbed on $\mathrm{ZnS}: \mathrm{Mn}$ nanoparticles and of sodium mercaptoacetate along with the DFT-calculated harmonic frequencies using B3LPY/6-31G(d). The vibrational assignment is based on the DFT calculations. In the DFT calculations, geometry optimization was perfonmed for a $\mathrm{Zn}$ ${ }^{-} \mathrm{SCH}_{2} \mathrm{CO}_{2}{ }^{-}$complex at the same level of theory and the same size of basis set. A scale factor, 0.9613, was used for vibrational frequencies

\begin{tabular}{|c|c|c|c|}
\hline \multicolumn{2}{|c|}{ ZnS:Mn-MAA Mercaptoacetate } & \multirow{2}{*}{$\begin{array}{l}\text { DFT } \\
256\end{array}$} & \multirow{2}{*}{$\frac{\text { Assignment }}{\mathrm{TO}(\mathrm{ZnS}) \text { or } v^{\prime}(\mathrm{Zn}-\mathrm{S})}$} \\
\hline $259 \mathrm{~s}$ & & & \\
\hline $347 \mathrm{~m}$ & & & $\mathrm{LO}(\mathrm{ZnS})$ \\
\hline $444 \mathrm{~m}$ & $432 \mathrm{~m}$ & 388 & $\delta(\mathrm{CCO}) / \rho(\mathrm{OCO})$ \\
\hline \multicolumn{4}{|l|}{$502 w$} \\
\hline $585 \mathrm{~m}$ & $563 \mathrm{vw}$ & 532 & $\gamma(\mathrm{CCO}) / \omega(\mathrm{OCO})$ \\
\hline $710 w$ & $660 \mathrm{vw}$ & 652 & $\delta(\mathrm{OCO})$ \\
\hline \multirow[t]{2}{*}{$787 \mathrm{~s}$} & $756 \mathrm{~m}$ & 784 & $v(\mathrm{CS})$ \\
\hline & $841 w$ & 844 & $v(\mathrm{CC})$ \\
\hline $932 \mathrm{~m}$ & $918 / 972 \mathrm{~m}$ & 863 & $\rho\left(\mathrm{CH}_{2}\right)$ \\
\hline $1170 \mathrm{w}$ & $1171 \mathrm{w}$ & 1183 & $\omega\left(\mathrm{CH}_{2}\right)$ \\
\hline \multirow[t]{2}{*}{$1398 \mathrm{~s}$} & $1385 \mathrm{~m}$ & 1305 & $v_{i s}(\mathrm{OCO})$ \\
\hline & $1418 \mathrm{~m}$ & 1419 & $\delta\left(\mathrm{CH}_{2}\right)$ \\
\hline \multirow[t]{2}{*}{$1597 w$} & $1616 \mathrm{nw}$ & 1658 & $v_{\text {as }}(\mathrm{OCO})$ \\
\hline & 2554 vs & & $v(\mathrm{SH})$ \\
\hline $2912 \mathrm{~s}$ & $2935 \mathrm{~s}$ & 2904 & $v_{i}\left(\mathrm{CH}_{2}\right)$ \\
\hline 2951 shoulder & $2974 \mathrm{~m}$ & 2955 & $v_{\text {as }}\left(\mathrm{CH}_{2}\right)$ \\
\hline
\end{tabular}

Abbreviations: $v$ stretch: $\delta$ in-plane bend: $\%$ out-of-plane bend: $\alpha$ rock: a) wag: TO. transverse optical phonon: LO, longitudinal optical phonon: fs, very strong; s. strong: m. medium: w. weak: vw, fery weak

$3 \mathrm{IG}(\mathrm{d})]^{1 \mathrm{ig}}$ using a primitive $\mathrm{Zn}-{ }^{-} \mathrm{SCH}_{2} \mathrm{CO}_{2}{ }^{-}$complex yield a $\mathrm{Zn}-\mathrm{S}$ stretching vibration of $256 \mathrm{~cm}^{-1}$. It is unlikely that the thiolate coordinates to Mn instead of $\mathrm{Zn}$ because very small amounts of Mn (only $3 \%$ of $\mathrm{Zn}$ ) were used. Raman spectral assignments based on the DFT calculations are listed in Table 1. Further calculations using a better model such as ${ }^{-} \mathrm{SCH}_{2} \mathrm{CO}_{2}{ }^{-}$on a two-dimensional $\mathrm{ZnS}$ surface are required to definitively assign the low frequency Raman region and to determine the structure of the adsorbate.

In conclusion. we have synthesized water-dispersible $\mathrm{ZnS}: \mathrm{Mn}$ nanocrystals by using MAA as a capping agent. Optical characterization shows that the prepared $\mathrm{ZnS}: \mathrm{Mn}$ MAA nanoparticles absorb at $307 \mathrm{~nm}$ and fluoresce at 570 nun. XRD and HR-TEM measurements reveal that the nano- crystals have a zinc-blende structure with a mean particle diameter of $3.8 \mathrm{~mm}$. Raman spectral analysis and DFT calculations suggest that MAA is deprotonated at both its thiol and carbosylic acid groups and that the thiolate group is bound to nanoparticle surfaces.

Acknowledgments. Prof. S. Yoon gratefully acknowledges the Dankook University research fund (2006).

Supporting Information. Detailed DFT calculation results are available via the internet at http: и' ww:kcsnet.or:kr bkcs.

\section{References}

1. Alivisatos. A. P. J. Phys. Chent. 1996. 100.13226

2. Murray. C. B.: Kagan. C. R.: Bawendi. M. G. Amu. Rev. Mater. Sci. 2000.30 .545 .

3. Bhargava, R. N.: Gallagher, D.: Hong. X.; Nurmikio, A. Plns. Rev Lett. 1994. 72, 416.

4. Gan. L. M.: Liu. B.: Chew C. H.: Xu. S. J.; Chua. S. J: Loy G. L: Xu. G. Q. Langnuir 1997. 13.6427.

5. Bol. A. A.: Meijerith. A. Phns Rev: B: Condens. Matter 1998. 58. R15997.

6. Xu. S. J: Chua, S. J.: Liu, B.: Gan, L. M.; Chew. C. H.; Xu. G. Q. Appl. Plys. Lett. 1998. 73, 478 .

7. Tanaka, M.: Sawai. S.; Sengoku, M; Kato. M; Masumoto. Y. d. Appl. Phys. 2000. 87. 8535.

8. Chent. W.: Su. F.: Li. G.: Toly. A. G.: Malm1. J-O.: Bovin. J-O. J A Apl. Phys. 2002.92. 1950.

9. Suyver. J. F.: Wuister, S. F.; Kelly, J. J.: Meijerink. A. Kano Lett. 2001. 1,429 .

10. Jun. Y. W. Jang, J. T.: Cheon, J. W. Bull Korean Chem. Soc. 2006. 27.961.

11. Chan1. W. C. W.: Nie. S. Science 1998. 281. 2016.

12. Li. X: Zhang. T.: Xu. W.: Tia. H.: Wang. X: Yang. B.: Zhao. B.: Li. B.: Ozaki, Y. Langmio 2003, 19,4285.

13. Zhuang, J.: Zhang. X.: Wang. G.: Li. D.: Yang, W.: Li, T. J. Hater: Chem 2003,13, 1853 .

14. Wang. Y.: Sun. Z.: Hu. H.: Jing. S.: Zhao. B.: Xu. W.: Zhao. C.: Lombardi. T. R. J. Raman Spectrose. 2007. 38. 34.

15. Castro. J. L.: Lopez-Ramirez. M. R.: Centeno. S. P.: Otero. J. C. Biopolymers 2004. 7f, 141 .

16. Ito. K.: Bernstein. H. J. Can. J. Chem. 1956, 34. 170.

17. Schneider. J.: Kirby, R. D. Plys. Rev B: Condens. Hatter 1972, 6. 1290 .

18. Yang. R. D.: Tripathy. S.: Tay. F. E. H.: Gan1. L. M.: Chua. S. J. J. Iac. Sci. Technol. B 2003. 2l. 984

19. Frisch. M. J.: Trucks. G. W: Schlegel. H. B.: Scuseria, G. E: Robb. M. A. et al. Gaussian 03. Revision C.02: Gaussian, Ine: Wallingford. CT, 2004. 